\title{
Differential Response of Dystonia and Parkinsonism following Globus Pallidus Internus Deep Brain Stimulation in X-Linked Dystonia-Parkinsonism (Lubag)
}

\author{
Genko Oyama $^{a}$ Hubert H. Fernandez ${ }^{a}$ Kelly D. Foote ${ }^{b}$ Pam Zeilman $^{a}$ \\ Nelson Hwynn ${ }^{a}$ Charles E. Jacobson, IV ${ }^{a}$ Irene A. Malaty ${ }^{a}$ \\ Ramon L. Rodriguez ${ }^{a}$ Michael S. Okun ${ }^{a}$ \\ Departments of a Neurology, and ${ }^{\mathrm{b}}$ Neurosurgery, University of Florida College of Medicine/Shands Hospital, \\ Movement Disorders Center, McKnight Brain Institute, Gainesville, Fla., USA
}

\section{Key Words}

X-linked dystonia-parkinsonism • Globus pallidus internus • Cognitive impairment $\cdot$ Medication-resistant parkinsonism

\begin{abstract}
Background: X-linked dystonia-parkinsonism (XDP; DYT3; Lubag) is an adult-onset hereditary progressive dystonia/ parkinsonism which is typically minimally responsive to pharmacological treatment. Case Report: We report a 63year-old man with a diagnosis of XDP who underwent bilateral globus pallidus internus deep brain stimulator (GPi-DBS) placement. His course initially began with right hand tremor and dystonia at age 57 and progressed to also include bradykinesia and rigidity. The patient tolerated the procedure without significant complications. GPi-DBS improved his right hand dystonia, but did not significantly improve his parkinsonism. Conclusion: DBS may be a therapeutic option for select cases of XDP, but its specific indications must be carefully discussed, as the available cases have had mixed responses. Whether other targets may be more effective is not known.

Copyright ๑ 2010 S. Karger AG, Basel
\end{abstract}

\section{Introduction}

X-linked dystonia-parkinsonism (XDP; DYT3; Lubag) is an adult-onset hereditary progressive disorder with dystonia and parkinsonism that primarily affects Filipino men from the island of Panay [1]. In many reported cases, it is often medication resistant or minimally responsive to pharmacologic treatment [2]. The disabling symptoms have also been surgically refractory in the limited available reports in the literature where thalamotomy was employed $[3,4]$. To date, there are two existing case reports on bilateral globus pallidus internus deep brain stimulation (GPi-DBS) to address XDP symptoms $[5,6]$. We report our experience with GPi-DBS in XDP, and we discuss the varying responses of parkinsonism and dystonia to DBS. We previously reported this patient's detailed preoperative clinical features and diffuse cognitive impairment [7].

\section{KARGER}

() 2010 S. Karger AG, Basel

Fax +41613061234

E-Mail karger@karger.ch

www.karger.com 


\section{Case Report}

The patient was a 63-year-old right-handed Filipino man, a native of the island of Panay, who was referred for treatment of medication-resistant parkinsonism and dystonia. His initial symptom was a right hand pill-rolling tremor at age 57. Gradually he developed increasing bradykinesia, rigidity and painful dystonic posturing involving the wrists and feet. Additionally, his ambulation progressively worsened. Two years later, his dystonic posturing in the right upper extremity had advanced to the extent that he had constant inward rotation of the right shoulder and involuntary flexion of the right wrist. He had a tendency to hold the right arm behind his body especially when walking. He was initially diagnosed with idiopathic Parkinson's disease by his primary neurologist, and his parkinsonian symptoms slightly responded to ropinirole treatment. He was treated with maximally tolerated doses of ropinirole, carbidopa/levodopa, and trihexyphenidyl; however, his symptoms continued to worsen. He eventually underwent genetic testing, which confirmed the diagnosis of XDP. The baseline Unified Parkinson's Disease Rating Scale Part 3 (UPDRS-III) score performed at our institution was 43 points, the Unified Dystonia Rating Scale (UDRS) score was 13 points, and Burke-FahnMarsden Dystonia Rating Scale (BFM-DRS) was 14 points (tested in his 'on' dopaminergic medication state).

His Dementia Rating Scale (DRS) score was 113/144, and he was found to have diffuse cognitive decline and mild depression. However, because his dystonia and parkinsonism were very disabling, greatly affecting his quality of life, our DBS surgical team as well as the patient and his wife ultimately decided it was in his best interest to undergo a DBS operation to potentially improve his quality of life. Microelectrode-guided bilaterally staged GPiDBS was performed without anesthesia (lidocaine for frame placement) with the right DBS lead implanted 14 days after the left. Microelectrode recordings (three passes on the left, two passes on the right) identified sensorimotor GPi, GPe, capsular border, and the optic tract. Macrostimulation was performed to confirm microelectrode recordings. He was implanted with Model 3387 DBS leads (Medtronic, Minneapolis, Minn., USA). The placement of the ventral electrode tips was confirmed postoperatively by CT-MRI fusion (left: $21.5 \mathrm{~mm}$ lateral, $4 \mathrm{~mm}$ anterior, 5.5 $\mathrm{mm}$ inferior; right: $23.0 \mathrm{~mm}$ lateral, $4.5 \mathrm{~mm}$ anterior, $4.5 \mathrm{~mm}$ inferior all relative to the midpoint of the AC-PC line (see fig. 1 showing lead reconstructions in lateral anterior GPi). One month following lead implantation, subclavicular generators (Soletra; Medtronic) were implanted and connected to his DBS leads under general anesthesia. The stimulators were activated 30 days following the initial surgery, and the thresholds for motor side effects of each DBS contacts were confirmed (utilizing a pulse width of $90 \mu \mathrm{s}$ and a rate of $135 \mathrm{~Hz}$; right: contact $0-4.5 \mathrm{~V}$, contact 1 $4.3 \mathrm{~V}$, contact $2-7.0 \mathrm{~V}$, contact $3-6.8 \mathrm{~V}$; left: contact $0-2.5 \mathrm{~V}$, contact $1-3.5 \mathrm{~V}$, contact $2-4.1 \mathrm{~V}$, contact $3-4.8 \mathrm{~V})$. His final stimulation parameters were $2(-) 3(-) \mathrm{C}(+), 3.3 \mathrm{~V}, 90 \mu \mathrm{s}, 160 \mathrm{~Hz}$ for the left DBS lead, and 2(-)C(+), 3.1 V, $90 \mu \mathrm{s}, 160 \mathrm{~Hz}$ for the right DBS lead. His disabling dystonic posturing of the distal right arm immediately improved and the benefit was sustained at 1-year follow-up (total UDRS 5, BFM-DRS 4.5; see table 1). However, his parkinsonism failed to respond in a clinically significant manner (UPDRS-III on medication/on stimulation was 43 , table 1). His tremor improved but his rigidity, bradykinesia, postural stability and gait did not change and might have overall slightly worsened.
He still tended to fall due to freezing of gait, and he continued to require assistance with standing as well as with some activities of daily living. Although his depression improved, his moderate dementia remained unchanged and his overall motivation slightly worsened. He and his wife appreciated dystonia and tremor improvement, but they were disappointed that gait did not improve despite preoperative counseling. They both agreed the improvement was substantial enough that if given the choice they would have elected to have the operation again.

\section{Discussion}

To our knowledge, there are two reported XDP cases treated by GPi-DBS. The features of these cases as well as our case are all summarized in table 1 . The first case reported by Evidente et al. [5] was a 45 -year-old man who first presented with shuffling gait at age 31 . He later developed handwriting difficulty, then lower limb dystonia, dysarthria with jaw opening dystonia, and facial tremor. One week after bilateral GPi-DBS implantation, his generalized dystonia improved and he had objective evidence of further improvement at subsequent programming sessions. At 1-year follow-up, his UPDRS and BFMDRS were all dramatically improved.

The second case reported by Martinez-Torres et al. [6] was a 34-year-old man with a 12-month history of a rapidly progressive dystonia. His initial symptom was blepharospasm, and subsequently, he developed generalized dystonia that included the arms, legs, trunk, neck (retrocollis), and oropharyngeal regions. After bilateral GPiDBS, he experienced an immediate improvement of dystonia even before switching the devices to an 'on' position. At 1-year follow-up, his trunk and oropharyngeal dystonia improved; however, his blepharospasm did not improve, and his retrocollis only partially responded.

Interestingly, all 3 cases experienced immediate improvement within 1 month, with one case reporting improvement of dystonia before the stimulators were turned on (Martinez-Torres's case). In our case, the improvement was evident immediately after starting stimulation, although lesional benefits cannot be ruled out.

Consistent with the prior reported cases as noted above, the dystonic component responded to GPi-DBS. However, a primary difference between our patient and the two reported cases was the lack of response of parkinsonian symptoms in our patient. Other differences were that previous XDP cases were of younger age at onset and had a more dystonia-predominant phenotype. Our patient had developed severe medication-refractory parkinsonism in addition to disabling dystonia. 

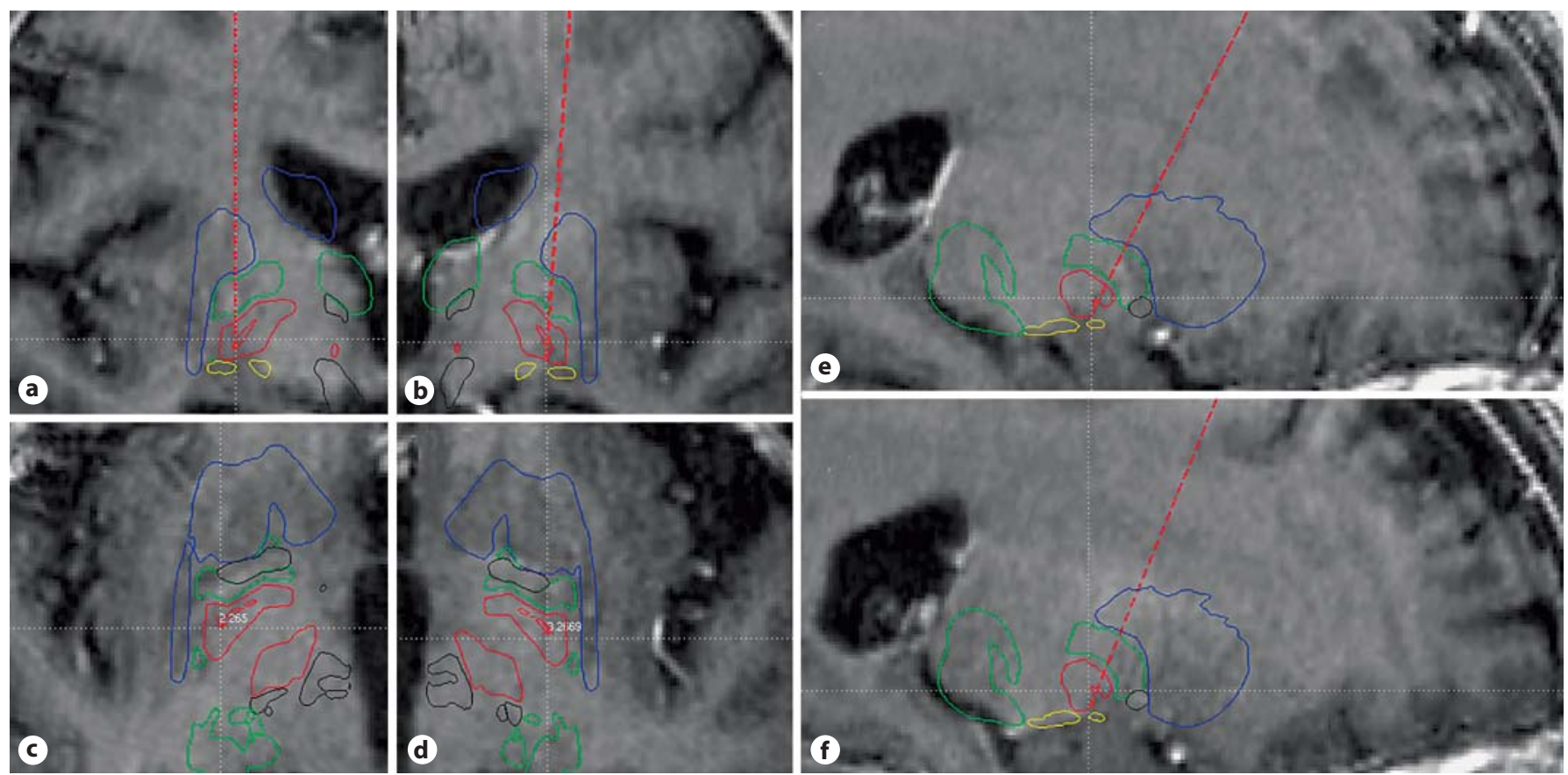

Fig. 1. $T_{1}$-weighted magnetic resonance images with superimposed atlas. The dotted line shows actual lead paths (calculated from lead location measured by postoperative CT): through right GPi (a) and left GPi (b) in coronal plane, right GPi (c) and left GPi (d) in axial plane, and right GPi (e) and left GPi (f) in sagittal plane.

Table 1. Clinical features and outcomes of GPi-DBS for XDP

\begin{tabular}{llll}
\hline & Evidente et al. [5] & Martinez-Torres et al. [6] & Present case \\
\hline Age at onset, years & 31 & 33 & 57 \\
\hline Sex & $\mathrm{M}$ & $\mathrm{M}$ & $\mathrm{M}$ \\
\hline Initial symptom & shuffling gait & blepharospasm & right hand tremor \\
\hline Subsequent symptoms & $\begin{array}{l}\text { handwriting difficulty, lower limb } \\
\text { dystonia, jaw opening dystonia }\end{array}$ & $\begin{array}{l}\text { generalized dystonia } \\
\text { parkinsonism (rigidity, } \\
\text { akinesia, gait disturbance) }\end{array}$ \\
\hline Age at operation, years & 45 & 34 & 66 \\
\hline Disease duration before DBS, years & 14 & 1 & 9 \\
\hline Pre-UPDRS (off med.) & 33 & 37 & 48 \\
\hline Pre-UPDRS (on med.) & 20 & $\mathrm{NA}$ & 43 \\
\hline Pre-BFM-DRS & 40 & 77.5 & 14 \\
\hline Post-UPDRS (off stim.) & 21 & $\mathrm{NA}$ & 43 \\
\hline Post-UPDRS (on stim.) & 8 & 8 & 43 \\
\hline Post-BFM-DRS (on stim.) & 9.5 & 15 & 4.5 \\
\hline Follow-up, months & 12 & 12 & 12 \\
\hline Latency (response to DBS) & immediate & immediate & immediate \\
\hline Improved symptom & generalized dystonia & trunk dystonia and swallowing & right limb dystonia \\
\hline UPDRS, \% improvement & 75.8 & 78.4 & 10.4 \\
\hline BFM-DRS, \% improvement & 76.3 & 80.6 & 67.9 \\
\hline Stimulation parameters & $5.0 \mathrm{~V}, 210 / 120 \mu s, 130 \mathrm{~Hz}$ & $3.3 \mathrm{~V}, 60 \mu s, 130 \mathrm{~Hz}$ & $3.3 / 3.1 \mathrm{~V}, 90 \mu s, 160 \mathrm{~Hz}$ \\
\hline
\end{tabular}


XDP most commonly presents with a focal dystonia. The dystonia often progresses, and later in the course of the syndrome parkinsonism usually appears [1]. Only $6 \%$ of patients in a large series from the Philippines initially presented with parkinisonian symptoms [1]. The parkinsonism-predominant phenotype may slightly respond to levodopa [8], while dystonia in XDP is only partially responsive to anti-dystonia therapy $[2,3]$. The exact underlying pathogenesis of XDP is not known. Recently, a mutation in the TATA-binding protein-associated factor-1 has been described $[9,10]$. Neuropathological and MRI studies have revealed minimal global atrophy, and a multifocal or mosaic pattern of astrocytosis which includes early involvement of the caudate and putamen. The pathology also revealed severe atrophy in the caudate and the putamen $[1,4]$.

Two compartments in the striatum have been described: the striosome compartment and matrix compartment $[11,12]$. Goto et al. [13] identified loss of calcineurinexpressing neurons with accompanying gliosis in the striosome compartment in the early stage of XDP. They proposed that selective neuronal death within the striatum might result in an imbalance in activity between the striosomal and matrix-based pathways. This alteration in basal ganglia circuits may result in the dystonic predominant features, and later in the disease, may lead to the parkinsonian predominant features. This nonuniform mottled degeneration pattern of the striatum may in some way impact the staging of XDP, and may impact the variable response of specific symptoms to GPi stimulation. However, it should be noted that this is still speculative.

Another explanation for the variable clinical outcomes of DBS for XDP may relate to levodopa responsiveness of parkinsonism and dystonic features. DBS in PD tends to have its greatest effect on levodopa-responsive symptoms [14], whereas in primary dystonia, response to medications does not predict response to DBS [15]. These general rules seem to potentially hold true for select cases of XDP, but again these observations will require confirmation.
Finally, postoperative measurement of lead location and approximation by three-dimensional modeling showed that the leads were situated in the lateral sensorimotor portion of the GPi, but were positioned slightly anterior to what we would predict to be the ideal location for GPi stimulation. Although the thresholds for side effects were reasonable, we cannot exclude the possibility that the lead location may have impacted the differences of response between dystonia and parkinsonism. Leads implanted for dystonia may be purposely placed more anteriorly than leads for Parkinson's disease. The reason for the more anterior location is to allow for wider pulse widths, and larger charge densities, both of which may be required in dystonia. If leads are too posterior, current may spread into the internal capsule motor pathways, thus limiting the potential for stimulation effectiveness. In our case, one potential reason for less response to parkinsonism may have been the relatively more anterior location of the DBS leads. However, the lowest thresholds that induced motoric side effects for each lead were 2.5 and $4.5 \mathrm{~V}$, respectively, and these data would suggest the leads were less anterior than they appeared on the atlas morph (fig. 1).

GPi-DBS may be useful in select cases of Lubag syndrome; however, we would argue that clinicians should carefully examine dystonic versus parkinsonian symptoms as these features may have a differential response to DBS and patients and their families need to be thoroughly counseled on the expectations of DBS outcomes. Also, whether the parkinsonian features respond to a different brain target (i.e. subthalamic nucleus) and may benefit from refinements of the procedure remains unknown.

\section{Acknowledgement}

We would like to acknowledge the support of Tyler's Hope for a Dystonia Cure Center of Excellence and The US National Parkinson Foundation Center of Excellence.

\section{References}

\footnotetext{
1 Lee LV, Maranon E, Demaisip C, Peralta O, Borres-Icasiano R, Arancillo J, Rivera C, Munoz E, Tan K, Reyes MT: The natural history of sex-linked recessive dystonia parkinsonism of Panay, Philippines (XDP). Parkinsonism Relat Disord 2002;9:29-38.

2 Lee LV, Kupke KG, Caballar-Gonzaga F, Hebron-Ortiz M, Muller U: The phenotype of the X-linked dystonia-parkinsonism syndrome. An assessment of 42 cases in the Phil-
}

ippines. Medicine (Baltimore) 1991;70:179187.

- 3 Lee LV, Pascasio FM, Fuentes FD, Viterbo GH: Torsion dystonia in Panay, Philippines. Adv Neurol 1976;14:137-151.

-4 Waters CH, Faust PL, Powers J, Vinters H, Moskowitz C, Nygaard T, Hunt AL, Fahn S: Neuropathology of Lubag (X-linked dystonia parkinsonism). Mov Disord 1993;8:387390. 
5 Evidente VG, Lyons MK, Wheeler M, Hillman R, Helepolelei L, Beynen F, Nolte D, Muller U, Starr PA: First case of X-linked dystonia-parkinsonism ('Lubag') to demonstrate a response to bilateral pallidal stimulation. Mov Disord 2007;22:1790-1793.

-6 Martinez-Torres I, Limousin P, Tisch S, Page R, Pinto A, Foltynie T, Bhatia KP, Hariz MI, Zrinzo L: Early and marked benefit with GPi DBS for Lubag syndrome presenting with rapidly progressive life-threatening dystonia. Mov Disord 2009;24:1710-1712.

7 Howe LL, Kellison IL, Fernandez HH, Okun MS, Bowers D: Neuropsychological profile of a Filipino gentleman with X-linked dystonia-parkinsonism: a case report of Lubag disease. Clin Neuropsychol 2009;23:100117.
Evidente VG, Gwinn-Hardy K, Hardy J, Hernandez D, Singleton A: X-linked dystonia ('Lubag') presenting predominantly with parkinsonism: a more benign phenotype? Mov Disord 2002;17:200-202.

$\checkmark 9$ Nolte D, Niemann S, Muller U: Specific sequence changes in multiple transcript system DYT3 are associated with X-linked dystonia parkinsonism. Proc Natl Acad Sci USA 2003;100:10347-10352.

10 Herzfeld T, Nolte D, Muller U: Structural and functional analysis of the human TAF1/ DYT3 multiple transcript system. Mamm Genome 2007;18:787-795.

11 Graybiel AM: Neurotransmitters and neuromodulators in the basal ganglia. Trends Neurosci 1990;13:244-254.
12 Gerfen CR: The neostriatal mosaic: multiple levels of compartmental organization in the basal ganglia. Annu Rev Neurosci 1992;15: 285-320.

$\checkmark 13$ Goto S, Lee LV, Munoz EL, Tooyama I, Tamiya G, Makino S, Ando S, Dantes MB, Yamada K, Matsumoto S, Shimazu H, Kuratsu J, Hirano A, Kaji R: Functional anatomy of the basal ganglia in X-linked recessive dystoniaparkinsonism. Ann Neurol 2005;58:7-17.

14 Rodriguez RL, Fernandez HH, Haq I, Okun MS: Pearls in patient selection for deep brain stimulation. Neurologist 2007;13:253-260.

15 Isaias IU, Alterman RL, Tagliati M: Outcome predictors of pallidal stimulation in patients with primary dystonia: the role of disease duration. Brain 2008;131:1895-1902. 